\title{
Aligners: The Science of Clear Orthodontics
}

Sunegha Kundal', Tulika Shokeen ${ }^{2}$

\author{
${ }^{1}$ Specialist Orthodontist, Private Practitioner, Jammu and Kashmir, India, ${ }^{2}$ Specialist Orthodontist, Private \\ Practitioner, Delhi, India
}

\begin{abstract}
Since their inception, clear aligners have undergone multitude of advancements that aimed to make the orthodontic treatment less conspicuous. Used for moving teeth for alignment and creating healthy and beautiful smiles, like many other orthodontic appliances, clear aligners have proven to be a much more comfortable and esthetic alternative. The treatment success relies on clinicians' knowledge and experience with the aligners, proper case selection, and the patients' adherence to the treatment protocols. The area of exploration of this review is limited to providing an overview of the clear aligner treatment including benefits and limitations, principles and biomechanics of clear aligner treatment, materials used, methods of fabrication, and wear time. Umpteen manufacturers have emerged accounting for the increased demand by patients seeking orthodontic treatment, with the number increasing every year. It is outside the purview of this article to elucidate and compare the array of clear aligner systems available today.
\end{abstract}

Key words: Esthetics, clear aligners, orthodontics, tooth movement

\section{INTRODUCTION}

Orthodontic treatment has long been synonymous to a mouth full of "unattractive" wires. The stigma extended beyond outer appearance, with people perceiving those with metal braces to have a lower intellect. ${ }^{[1]}$ Introduction of clear aligners thus came as a respite to the orthodontic patient, for their esthetic superiority made them more attractive to metal and ceramic brackets. ${ }^{[2]}$ These are the active clear plastic trays fitting snugly onto the teeth, each worn for 2 weeks on an average, and changed sequentially to accomplish the incorporated tooth movements. From being used for mild malocclusion cases at the outset to treating vast variety of cases contemporarily, from minimal crowding to bicuspid extractions, clear aligners have come a long way and still continue to evolve.

\begin{tabular}{|l|l|}
\hline \multicolumn{2}{|c|}{ Access this article online } \\
\hline \multicolumn{1}{|c|}{ Publisher } & $\begin{array}{l}\text { Website: } \\
\text { www.ijdms.in }\end{array}$ \\
\hline & DOI: 10.30954/IJDMS.1.2020.9 \\
\hline
\end{tabular}

\section{THE ORIGIN STORY}

Contrary to popular belief, clear aligners are not a new find. The chronology leading up to their present-day use dates to 1945 , when Dr. Harold D. Kesling first advocated a rubber appliance for moving teeth. ${ }^{[3]}$ In 1964, Nahoum fabricated the dental contour appliance in what was to be the first thermoformed plastic sheet for moving teeth. ${ }^{[4]}$ Sheridan modified the same in 1993, calling it the Essix Appliance. ${ }^{[5]}$ However, fabricating the appliance was a laborious process requiring impression-making at every successive appointment. The solution arrived in the form of a clear aligner system created digitally by two Stanford graduates - Zia Chishti and Kelsey Wirth. Called Invisalign ${ }^{\circledR}$, it was launched in 1997 by Align Technology $@$ (Santa Clara, CA) and is credited with bringing a comfortable alternative to braces to mainstream orthodontics. In 2005 , a competitor emerged in the form of OrthoClear ${ }^{\circledR}-$ a clear aligner system developed independently by one of the original founders Zia Chishti. However, it was withdrawn a year later due to lawsuits alleging patent infringement. Many practicing clear aligner users suffered as a result, one of whom was Dr. Willis Pumphrey whose 400 patients were stranded mid-treatment. He responded by developing ClearCorrect

\section{Address for Correspondence:}

Dr. Sunegha Kundal, Specialist Orthodontist, Private Practitioner, Jammu - 180011, Jammu and Kashmir, India. Phone: +919086505505. E-mail:suneghakundal@gmail.com

Submission: 27 May 2020; Revision: 16 June 2020; Acceptance: 28 June 2020 
(ClearCorrect, Round Rock, TX, USA), an Invisalign ${ }^{\circledR}$ alternative that quickly gained popularity. An onslaught of clear aligner systems has since followed that are being used to correct minor to complex malocclusions. ${ }^{[6]}$

\section{BENEFITS AND LIMITATIONS}

\section{Benefits}

i. Esthetics: A transparent, clear design that is discreet.

ii. Comfort: Free from brackets or wires and resultant cuts and ulcerations.

iii. Improved oral hygiene and periodontal health: Removable nature allows proper brushing and flossing.

iv. No food restrictions: Patients are free to eat or drink anything during clear aligner therapy (CAT).

v. Predictable treatment time: Digital planning ensures a precise estimation of treatment time.

vi. Decreased dental office visits: Patients themselves change their subsequent aligners, requiring fewer office visits.

vii. Minimal emergencies: Significantly reduced emergency appointments with CAT as compared to fixed orthodontic treatment.

viii.Possible to include teeth with structural anomalies and difficult-to-bond surfaces.

\section{Limitations}

i. Dependency on patient compliance: A wear time of 22 $\mathrm{h} /$ day is mandatory for therapy to be effective.

ii. Limited extent of tooth movements by aligners alone: For complex movements, auxiliaries are required as an adjunct to aligners.

iii. Higher cost.

iv. Initial slurring of speech: Subsides subsequently after 2-3 days of wear.

v. Breakages: While wearing or removing if the patient applies excessive force.

vi. Chances of losing the aligners: Removable nature makes aligners prone to being misplaced.

vii. Inconvenience: Removing aligners every time one eats or drinks can be a burden.

viii.Manufacturing defects: Results in ill-fitting aligners.

\section{PRINCIPLES OF CAT}

CAT works on two basic principles: ${ }^{[7]}$

1. Creating space: Through arch expansion, tooth extraction, or interproximal reduction (IPR).

2. Force/pressure application: Through small incremental movements incorporated in aligner itself and with auxiliaries (attachments, dimples, elastics, or digital power chains). The amount of pressure required for moving a tooth depends on factors such as tooth shape, tooth size, type of movement, and periodontal condition.

\section{BIOMECHANICS OF CAT}

The mechanism of tooth movement with clear aligners is centered around two systems: ${ }^{[8,9]}$

1. Displacement-driven system: Controls tipping and minor rotations. Aligners are fabricated as per next staged position; the tooth continues to move till the aligner becomes passive. No root movement elicited.

2. Force-driven system: The software determines the type of movement required for an individual tooth, the mechanical principles needed to achieve that movement, and aligner shape. Pressure points and attachments are incorporated into aligners that apply the forces required for planned movements.

\section{TYPE OF MATERIALS USED}

Clear aligner materials have evolved from a single layered or monophasic plastic to the $2^{\text {nd }}$ generation polyurethane material, to the currently used $3^{\text {rd }}$ generation multilayered polyurethane-like material that comprises of hard and soft layers. While the soft layer imparts the property of elastic deformation allowing smooth seating of the aligner, the hard layer ensures strength and durability. ${ }^{[10]}$ Polyethylene terephthalate glycol modified (PET-G) remains the commonly used material. Other materials include polypropylene, polycarbonate, thermoplastic polyurethanes, and ethylene vinyl acetate. ${ }^{[1]}$ Table 1 enlists popular clear aligner brands and the material used for aligner fabrication. ${ }^{[12]}$

Table 1: Clear aligner brands and the material used by them for aligner fabrication

\begin{tabular}{|c|c|c|c|c|}
\hline Code & Thickness & $\begin{array}{l}\text { Product } \\
\text { name }\end{array}$ & Manufacture & $\begin{array}{l}\text { Component } \\
\text { (MSDS) }\end{array}$ \\
\hline EVA & $1.0 \mathrm{~mm}$ & Bioplast & $\begin{array}{l}\text { Scheu-Dental, } \\
\text { GmbH }\end{array}$ & $\begin{array}{l}\text { Ethylene- } \\
\text { vinyl acetate } \\
\text { copolymer }\end{array}$ \\
\hline PE & $1.0 \mathrm{~mm}$ & Copyplast & $\begin{array}{l}\text { Scheu-Dental, } \\
\text { GmbH }\end{array}$ & Polyethylene \\
\hline PETG & $1.0 \mathrm{~mm}$ & Duran & $\begin{array}{l}\text { Scheu-Dental, } \\
\text { GmbH }\end{array}$ & $\begin{array}{l}\text { Polyethylene } \\
\text { terephthalate } \\
\text { glycol }\end{array}$ \\
\hline PP & $0.8 \mathrm{~mm}$ & Hardcast & $\begin{array}{l}\text { Scheu-Dental, } \\
\text { GmbH }\end{array}$ & Polypropylene \\
\hline $\mathrm{PC}$ & $0.75 \mathrm{~mm}$ & $\begin{array}{l}\text { Imprelon } \\
\text { "S" }\end{array}$ & $\begin{array}{l}\text { Scheu-Dental, } \\
\text { GmbH }\end{array}$ & Polycarbonate \\
\hline$A+$ & $0.040 \mathrm{in}$. & Essix A+ & $\begin{array}{l}\text { Raintree } \\
\text { Essix, Inc. }\end{array}$ & Copolyester \\
\hline $\mathrm{C}+$ & $0.040 \mathrm{in.}$ & Essix C+ & $\begin{array}{l}\text { Raintree } \\
\text { Essix, Inc. }\end{array}$ & $\begin{array}{l}\text { Polypropylene/ } \\
\text { ethylene } \\
\text { copolymer } \\
(>95 \%), \\
\text { stabilizers (<5\%) }\end{array}$ \\
\hline PUR & $0.030 \mathrm{in.}$ & Invisalign & $\begin{array}{l}\text { Align } \\
\text { Technology, } \\
\text { Inc. }\end{array}$ & $\begin{array}{l}\text { Polyurethane } \\
\text { from methylene } \\
\text { diphenyl } \\
\text { diisocyanate and } \\
1,6 \text {-hexanedial, } \\
\text { Additives }\end{array}$ \\
\hline
\end{tabular}

Component as obtained from the manufactures' material safety data sheet 


\section{PROPERTIES OF CLEAR ALIGNER MATERIAL}

\section{Mechanical Properties}

Force delivery of clear aligners depends on:

a. Amount of activation: The extent of movement prescribed per aligner differs for every clear aligner system. ClearSmile ${ }^{\circledR}$ system allows $0.5 \mathrm{~mm}$, ClearCorrect (ClearCorrect, Round Rock, TX, USA) prescribes $0.3 \mathrm{~mm}$, whereas Invisalign (Align Technology, Inc., Santa Clara, CA, USA) incorporates $0.25 \mathrm{~mm}$ of tooth movement per aligner. ${ }^{[13,14]}$

b. Type of material: Pressure formed appliance exerts greater force systems at higher rates of activation as compared to vacuum formed ones. ${ }^{[15,16]}$

c. Thickness of material: Ranges from 0.5 to $1.5 \mathrm{~mm}$; thicker the material, higher the force. ${ }^{[17,18]}$

d. Size and shape of adjacent teeth. ${ }^{[16]}$

Wear resistance of an aligner is indicated by the hardness of its material; higher the hardness value, better the wear resistance. Similarly, higher elastic index indicates brittleness of the material; a high modulus of elasticity increases the force delivery capacity under constant strain, which is a desirable property for an aligner material. ${ }^{[19]}$

\section{Aging}

Mechanical properties of the aligner material deteriorate with time. Microcracks, delaminated areas, calcified biofilm deposits, and loss of transparency were reported in Invisalign (Align Technology, Inc., Santa Clara, CA, USA) aligners worn for 2 weeks. ${ }^{[20,21]}$ The residual stresses decrease in the thermoplastic materials with time. ${ }^{[22]} \mathrm{A}$ change in fit of the appliance and resultant orthodontic forces can be seen as a result of intraoral hygroscopic expansion. Water absorption of thermoplastic materials increases with time, with Invisalign (Align Technology, Inc., Santa Clara, CA, USA) showing the highest absorption followed by PET-G. ${ }^{[12]}$

\section{Thermal Properties}

All polymers have a glass-transition temperature $\left(\mathrm{T}_{\mathrm{g}}\right)$ at which the rigid state converts to a rubbery state. When thermal properties of three clear aligners (Invisalign [Align Technology, Inc., Santa Clara, CA, USA), Simpli5 [Allesee Orthodontic Appliances, Sturtevant, WI, USA], and ClearCorrect [ClearCorrect, Round Rock, TX, USA]) were compared, all three aligner systems were found to have a $\mathrm{T}_{g}$ above the accepted oral maximum temperature, with no significant difference seen before and after clinical use. ${ }^{[23]}$

\section{Stainability}

The aligner material should be able to maintain its color stability and transparency. To avoid pigment adsorption, it is recommended to remove aligners during eating and while drinking colored drinks. Color changes in aligners have been reported on exposure to staining solutions such as coffee, black tea, and red wine. ${ }^{[24]}$ Invisalign (Align Technology, Inc., Santa Clara, CA, USA) was more prone to pigmentation when exposed to coffee or red wine. ${ }^{[25]}$

\section{FABRICATION OF CLEAR ALIGNERS}

\section{Manual}

The individual teeth are sectioned and repositioned sequentially using a wax setup. Once a working cast is obtained, teeth are removed from cast and moved into the planned position, with aligners sheets molded onto the realigned models using pressure molding or vacuum machine. ${ }^{[26]}$

\section{Digital (CAD-CAM) $)^{[27]}$}

- Patients' impressions obtained digitally by intraoral scanner and uploaded for virtual treatment planning.

- In case of traditional impressions (polyvinyl siloxane putty), casts are obtained, scanned, and uploaded.

- Digital models are then analyzed for IPR and expansion requirements.

- Teeth are sectioned digitally.

- IPR prescription is reviewed for validity and if acceptable, IPR is performed digitally and plan is communicated to the doctor.

- Teeth are then moved into correct alignment digitally and a digital overlay model is created by superimposing final model onto the original model.

- Sequence of aligners fabricated using the overlay model.

- The force bumps, attachments, or auxiliaries are then planned for aiding tooth movements.

\section{GINGIVAL MARGIN DESIGN OF ALIGNERS}

Clear aligner should seat completely on insertion and be properly anchored to the dental arch without any dislodgement or vertical lifting. A decline in aligner forces at gum line is seen due to flexibility of gingival margins, thus impeding the ability to produce certain tooth movements. The possible margin designs are scalloped, straight cut at the gingival zenith, and straight cut $2 \mathrm{~mm}$ above the gingival zenith (most retentive) [Figure 1]. ${ }^{[28]}$ 


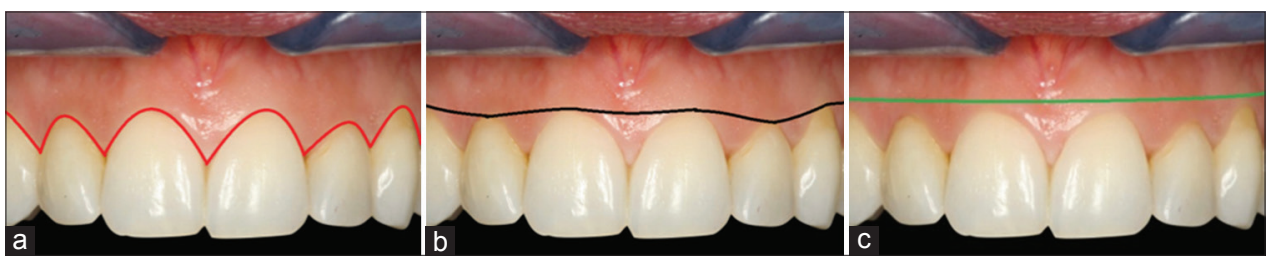

Figure 1: Gingival margin design of aligners: (a) Scalloped, (b) straight cut at gingival zenith, (c) straight cut 2 mm above the gingival zenith

\section{WEAR TIME OF ALIGNERS}

Wearing an aligner for $22 \mathrm{~h} /$ day for 2 weeks is mandated to ensure maximum expression of aligner prescription. ${ }^{[29]} \mathrm{A}$ decrease in daily wear time likely decreases the efficacy of certain incisor movements, reportedly labial inclination and buccolingual translation of mandibular incisors, rotation of maxillary central incisors, as well as buccolingual translation and intrusion of maxillary lateral incisors. ${ }^{[30]}$ However, most incorporated movements occur within the $1^{\text {st }}$ week, thus casting doubt over usefulness of the additional 1 week of aligner wear. ${ }^{[9,31]}$

\section{TOOTH MOVEMENTS WITH CLEAR ALIGNERS}

Replacing braces with clear polyurethane trays for orthodontic treatment have raised questions regarding its efficiency in moving the teeth. Tooth movement obtained most accurately with clear aligners is retrusion, followed by rotation, fan-type expansion, and protrusion, respectively. ${ }^{[32]}$ Acceptable results can be achieved for the buccolingual inclination of maxillary and mandibular incisors in mild-tomoderate malocclusions. ${ }^{[33]}$

CAT is recommended in non-extraction cases with mildto-moderate malocclusions in non-growing patients. ${ }^{[34]}$ In extraction cases, proper root angulations can be attained with use of suitable attachments and adequate knowledge of the system. ${ }^{[35]}$ Maxillary molar distalization $(2.5 \mathrm{~mm})$ and premolar extraction space closure $(7 \mathrm{~mm})$ are the most predictable and controlled movements that can be obtained. ${ }^{[36]}$ To its demerit, as occlusal contacts are lost due to thickness of aligners, problems with the final settling of the occlusal plane can arise. ${ }^{[37]}$

\section{ROOT RESORPTION WITH CLEAR ALIGNERS}

While fixed orthodontic appliances cause root resorption as a side effect of heavy orthodontic forces, there is no clear consensus on root resorption with CAT. A lower incidence and severity of root resorption has been reported, with incisors being the most affected teeth. ${ }^{[38]}$ The incidence of root resorption was seen to be comparable between aligners and light orthodontic forces. ${ }^{[39]}$ Another study revealed a lower severity and prevalence of root resorption with aligners. ${ }^{[40]}$

\section{CONCLUSION}

Clear aligner treatment has flourished since its introduction into orthodontics. Continued developments on the technological front are being adapted to improve its efficiency, especially in complex cases. Being a removable appliance, patient compliance is the foremost criteria for success of treatment with aligners and patient motivation is indispensable to avail the planned treatment outcomes. Arrays of companies are manufacturing aligners today, providing them directly to consumers, or routing through the dentists; it falls beyond the scope of this article to study the individual characteristics of each system.

\section{REFERENCES}

1. Jeremiah HG, Bister D, Newton JT. Social perceptions of adults wearing orthodontic appliances: A cross-sectional study. Eur J Orthod 2011;33:476-82.

2. Ziuchkovski JP, Fields HW, Johnston WM, Lindsey DT. Assessment of perceived orthodontic appliance attractiveness. Am J Orthod Dentofacial Orthop 2008;133:S68-78.

3. Kesling HD. The philosophy of the tooth positioning appliance. Am J Orthod 1945;31:297-304.

4. Nahoum $\mathrm{H}$. The vacuum formed dental contour appliance. N Y State Dent J 1964;9:385-90.

5. Sheridan JJ, LeDoux W, McMinn R. Essix retainers: Fabrication and supervision for permanent retention. J Clin Orthod 1993;27:37-45.

6. Available from: https://www.riversidedental.com.au/patientresources/academic-articles/history-of-aligners. [Last accessed on 2020 Jul 21].

7. Available from: https://www.support.clearcorrect.com/hc/ en-us/articles/115006636087-2-basic-principles-for-clear-alignertreatment.

8. Tamer I, Öztaş E, Marşan G. Orthodontic treatment with clear aligners and the scientific reality behind their marketing: A literature review. Turk J Orthod 2019;32:241-6.

9. Drake CT, McGorray SP, Dolce C, Nair M, Wheeler TT. Orthodontic tooth movement with clear aligners. ISRN Dent 2012;2012:657973.

10. Shotell MD. The proliferation of clear aligner orthodontics: Workflows, materials, and designs. Compend Contin Educ Dent 2020;41:340-41.

11. Dupaix RB, Boyce MC. Finite strain behavior of poly (ethylene terephthalate) (PET) and poly (ethylene terephthalate)-glycol (PETG). Polymer 2005;46:4827-38.

12. Ryokawa H, Miyazaki Y, Fujishima A, Miyazaki T, Maki K. The 
mechanical properties of dental thermoplastic materials in a simulated intraoral environment. Orthod Waves 2006;65:64-72.

13. Simon M, Keilig L, Schwarze J, Jung BA, Bourauel C. Forces and moments generated by removable thermoplastic aligners: Incisor torque, premolar derotation, and molar distalization. Am J Orthod Dentofacial Orthop 2014;145:728-36.

14. Boyd RL. Predictability of successful orthodontic treatment using Invisalign, The Greater Philadelphia Society of Orthodontists page. Santa Clara, CA, USA: Align Technology, Inc.; 2003. Available from: http://www.gpso.org/events/2003_outline.pdf. [Last accessed on 2020 Jul 21].

15. Hahn W, Dathe H, Fialka-Fricke J, Fricke-Zech S, Zapf A, KubeinMeesenburg D, et al. Influence of thermoplastic appliance thickness on the magnitude of force delivered to a maxillary central incisor during tipping. Am J Orthod Dentofacial Orthop 2009;136:12. e1-13.

16. Hahn W, Engelke B, Jung K, Dathe H, Fialka-Fricke J, KubeinMeesenburg D, et al. Initial forces and moments delivered by removable thermoplastic appliances during rotation of an upper central incisor. Angle Orthod 2010;80:239-46.

17. Sifakakis I, Zinelis S, Eliades T. Aligners for orthodontic applications. In: Orthodontic Applications of Biomaterials. Cambridge: Woodhead Publishing; 2017. p. 275-85.

18. Kohda N, Iijima M, Muguruma T, Brantley WA, Ahluwalia KS, Mizoguchi I. Effects of mechanical properties of thermoplastic materials on the initial force of thermoplastic appliances. Angle Orthod 2013;83:476-83.

19. Alexandropoulos A, Al Jabbari YS, Zinelis S, Eliades T. Chemical and mechanical characteristics of contemporary thermoplastic orthodontic materials. Aust Orthod J 2015;31:165-70.

20. Schuster S, Eliades G, Zinelis S, Eliades T, Bradley TG. Structural conformation and leaching from in vitro aged and retrieved invisalign appliances. Am J Orthod Dentofacial Orthop 2004;126:725-8.

21. Gracco A, Mazzoli A, Favoni O, Conti C, Ferraris P, Tosi G, et al. Short-term chemical and physical changes in invisalign appliances. Aust Orthod J 2009;25:34-40.

22. Fang D, Zhang N, Chen H, Bai Y. Dynamic stress relaxation of orthodontic thermoplastic materials in a simulated oral environment. Dent Mater J 2013;32:946-51.

23. Louis W. Thermal Properties of Commonly Used Clear Aligner Systems As-Received and After Clinical Use, Master's Thesis, 2009; 2017. p. 431.

24. Liu CL, Sun W'T, Liao W, Lu WX, Li QW, Jeong Y, et al. Colour stabilities of three types of orthodontic clear aligners exposed to staining agents. Int J Oral Sci 2016;8:246-53.

25. Bernard G, Rompré P, Tavares JR, Montpetit A. Colorimetric and spectrophotometric measurements of orthodontic thermoplastic aligners exposed to various staining sources and cleaning methods. Head Face Med 2020;16:2.

26. Lagravère MO, Flores-Mir C. The treatment effects of invisalign orthodontic aligners: A systematic review. J Am Dent Assoc 2005;136:1724-9.
27. Anderson MC, Clatt BH, Hilliard JK, Julié D. Orthodontic Aligner Fabrication by Overlay Method. United States, US9, 345, 557B7; 2016.

28. Cowley DP, Mah J, O’Toole B. The effect of gingival-margin design on the retention of thermoformed aligners. J Clin Orthod 2012;46:697-705.

29. Joffe L. Invisalign: Early experiences. J Orthod 2003;30:348-52.

30. Carroll KM. Investigation into the Significance of Daily Invisalign Tray Wear Duration, Thesis. United States: The Ohio State University; 2018. Available from: https://www.etd.ohiolink. edu/!etd.send_file?accession=osu1522789295620812\&dispositio $\mathrm{n}=$ inline. [Last accessed on $2020 \mathrm{Jul} 22]$.

31. Bollen AM, Huang G, King G, Hujoel P, Ma T. Activation time and material stiffness of sequential removable orthodontic appliances. Part 1: Ability to complete treatment. Am J Orthod Dentofacial Orthop 2003;124:496-501.

32. Yildirım A. A Prospective Study to Determine the Efficiency of Clear Aligner Appliance, Doctoral Dissertation. Turkey: Istanbul University Institute of Health Sciences Orthodontics Department; 2013.

33. Rossini G, Parrini S, Deregibus A, Castroflorio T. Controlling orthodontic tooth movement with clear aligners: An updated systematic review regarding efficacy and efficiency. J Aligner Orthod 2017;1:7-20.

34. Robertson L, Kaur H, Fagundes NC, Romanyk D, Major P, Mir CF. Effectiveness of clear aligner therapy for orthodontic treatment: A systematic review. Orthod Craniofac Res 2020;23:133-42.

35. Papadimitriou A, Mousoulea S, Gkantidis N, Kloukos D. Clinical effectiveness of invisalign ${ }^{\circledR}$ orthodontic treatment: A systematic review. Prog Orthod 2018;19:37.

36. Li W, Wang S, Zhang Y. The effectiveness of the invisalign appliance in extraction cases using the the $\mathrm{ABO}$ model grading system: A multicenter randomized controlled trial. Int J Clin Exp Med 2015;8:8276-82.

37. Ke Y, Zhu Y, Zhu M. A comparison of treatment effectiveness between clear aligner and fixed appliance therapies. BMC Oral Health 2019;19:24.

38. Gay G, Ravera S, Castroflorio T, Garino F, Rossini G, Parrini S, et al.

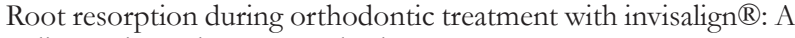
radiometric study. Prog Orthod 2017;18:12.

39. Zheng M, Liu R, Ni Z, Yu Z. Efficiency, effectiveness and treatment stability of clear aligners: A systematic review and meta-analysis. Orthod Craniofac Res 2017;20:127-33.

40. Li Y, Deng S, Mei L, Li Z, Zhang X, Yang C, et al. Prevalence and severity of apical root resorption during orthodontic treatment with clear aligners and fixed appliances: A cone beam computed tomography study. Prog Orthod 2020;21:1.

How to cite this article: Kundal S, Shokeen T. Aligners: The Science of Clear Orthodontics. Int J Dent Med Spec 2020;7(1):38-42.

Source of Support: None; Conflicts of Interest: None 